\title{
Le rappresentazioni degli italiani regionali: esperienze di formazione alla ricerca linguistica
}

Etna Rosa Krakenberger, Silvia Natale e Luisa Revelli

\section{(2) OpenEdition}

Edizione digitale

URL: https://journals.openedition.org/esp/4604

DOI: $10.4000 /$ esp.4604

ISSN: 2532-0319

Editore

Centre d'Information sur l'Éducation Bilingue et Plurilingue

\section{Edizione cartacea}

Data di pubblicazione: 1 décembre 2019

Paginazione: 63-68

ISSN: 1127-266X

\section{Notizia bibliografica digitale}

Etna Rosa Krakenberger, Silvia Natale e Luisa Revelli, «Le rappresentazioni degli italiani regionali: esperienze di formazione alla ricerca linguistica», Éducation et sociétés plurilingues [Online], 47 | 2019, Messo online il 01 septembre 2021, consultato il 02 septembre 2021. URL: http://

journals.openedition.org/esp/4604 ; DOI: https://doi.org/10.4000/esp.4604 


\title{
LE RAPPRESENTAZIONI DEGLI ITALIANI REGIONALI: ESPERIENZE DI FORMAZIONE ALLA RICERCA LINGUISTICA
}

\author{
Etna Krakenberger, Silvia Natale, Luisa Revelli (1)
}

Cette contribution présente une initiative inter-universitaire de formation à la recherche linguistique, mise en place conjointement par l'Institut de Langue et littérature italienne de l'Université de Berne et le Département des Sciences Humaines et Sociales de l'Université de la Vallée d'Aoste. La recherche porte sur les italiens régionaux, observés à travers les réactions qu'ils déclenchent chez les locuteurs. Le sujet des attitudes linguistiques se prête à l'analyse grâce à des activités de groupe visant à expérimenter plusieurs approches méthodologiques. La mise en place de ces activilés dans les deux universités se révèle une alternative à la dimension exclusivement individuelle que l'on associe encore trop souvent à la didactique et à la recherche universitaires.

Mots-clés: méthodologies, recherche linguistique, didactique universitaire, italiens régionaux, attitudes linguistiques

Der Beitrag stellt eine interuniversitäre Initiative innerhalb der Ausbildung der Sprachforschung vor, die gemeinsam vom Institut für italienische Sprache und Literatur der Universität Bern und dem Departement für Human- und Sozialwissenschaften der Universität des Aostatals initiiert wurde. Die Aktivität konzentriert sich auf das Thema des Regionalitalienischen bzw. auf die Reaktionen, die es bei Sprechern auslösen kann. Das Thema der Spracheinstellungen eignet sich für Gruppenanalysen, die darauf abzielen, mit unterschiedlichen methodischen Ansätzen zu experimentieren. Die gemeinsame Durchführung von Aktivitäten der beiden Universitäten wird als eine alternative Erfahrung zum Selbststudiums konzipiert, das nach wie vor sowohl in der universitären Didaktik als auch in der Forschung innerhalb der Geisteswissenschaften überwiegt.

Schlagwörter: Melhoden der Sprachforschung; Hochschuldidaklik, Regionalitalienisch, Spracheinstellung

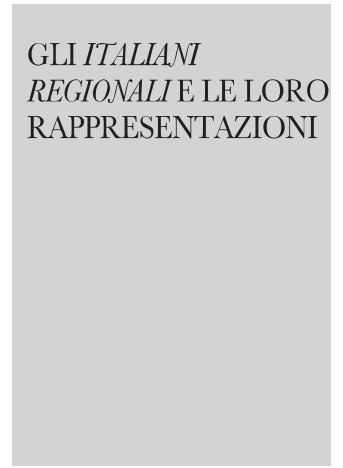

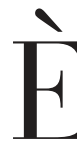

ben noto che, per ragioni connesse alla sua storia, l'italiano impiegato all'interno e all'esterno dei confini della Penisola si articola ancora oggi in una moltitudine di varietà caratterizzate da tratti latamente regionali (Telmon 1990) e che tali varietà geografiche sono sottoposte da parte dei parlanti, in maniera più o meno esplicita e consapevole, a valutazioni che - oltre ad assumere importanti implicazioni sotto il profilo relazionale psico-sociale possono condizionare gli apprendimenti linguistici, incidere sull'idea di norma linguistica e sulle sue applicazioni (Antonini \& Moretti 2000), intervenire nei processi di standardizzazione (De Pascale \& Marzo 2016) e avere effetti sulla stessa vitalità delle singole varietà (Catricalà, M., \& Di Ferrante, L. 2011). La letteratura scientifica 


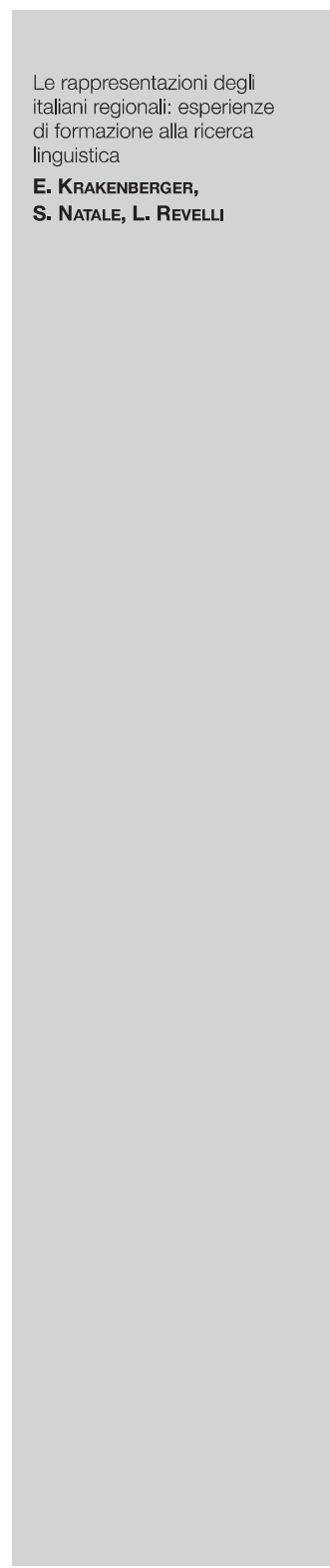

dedicata alle immagini degli italiani regionali centra il proprio focus sull'identificazione degli atteggiamenti dei parlanti, oggi piuttosto unanimemente intesi come posizioni concettuali pregiudiziali. Meno definito, per lo meno in relazione all'italiano, appare lo studio dei tratti su cui i parlanti comuni tendono a poggiare questi loro atteggiamenti e cioè dei segnali linguistici che individuano come marcatori di una determinata varietà locale da associare conseguentemente a valori positivi o negativi: il riconoscimento è generalmente ricondotto a questioni di accento e pronuncia, di cadenza e inflessione, di struttura melodica o andamento intonativo, ma i parlanti riescono raramente ad attribuire le varietà geografiche a località effettivamente pertinenti c circostanziate, ricorrendo più frequentemente a valutazioni impressionistiche, generiche e stereotipate, per esempio con approssimativo riferimento a macroaree (pronunce meridionali), con ricorso a processi di sovraestensione (napoletano per qualunque varietà meridionale) o fenomeni di scambio (napoletano per romano).

È sulla base di queste premesse che l'Istituto di Lingua e letteratura italiana dell'Università di Berna e il Dipartimento di Scienze Umane e Sociali dell'Università della Valle d'Aosta hanno concordato di attivare congiuntamente per l'anno accademico 2019/2020 un Seminario di formazione alla ricerca rivolto a gruppi di studenti delle due sedi e finalizzato ad indagare il tema degli italiani regionali e delle loro rappresentazioni.

Il Seminario circoscrive il proprio ambito d'interesse all'oralità e ancor più specificamente all'area fonologico-intonativa: le iniziative di innesco delle attività seminariali partono, infatti, da un questionario che prevede l'impiego di sette tracce orali fornite da parlanti rappresentativi di altrettante provenienze geografiche (Ticino, Valle d'Aosta, Milano, Firenze, Roma, Napoli, Palermo). Tutte le voci sono state prestate da uomini di mezza età ai quali è stato chiesto di leggere un testo senza introdurre tentativi di emendamento né di enfasi dei tratti regionali: l'impiego di voci diverse, che costituisce una diffusa variante alla tecnica classica del matched guise, si associa quindi alla lettura di un medesimo testo, composto da 52 parole e selezionato per la sua neutralità lessicale e sintattica. Alle sette letture diatopicamente marcate si aggiunge un'ottava traccia, quanto più possibile corrispondente all'italiano standard ovvero a una pronuncia neutra.

Si presume che le varietà selezionate possano risultare più o meno facilmente riconoscibili - oltre che in rapporto alla popolarità delle singole varietà nel sapere linguistico comune - sulla base di variabili individuali quali età, area di provenienza, distanza geografica, esperienze culturali e competenze metalinguistiche degli informatori.

Con l'obiettivo di verificare tali presupposti e di sperimentare un 


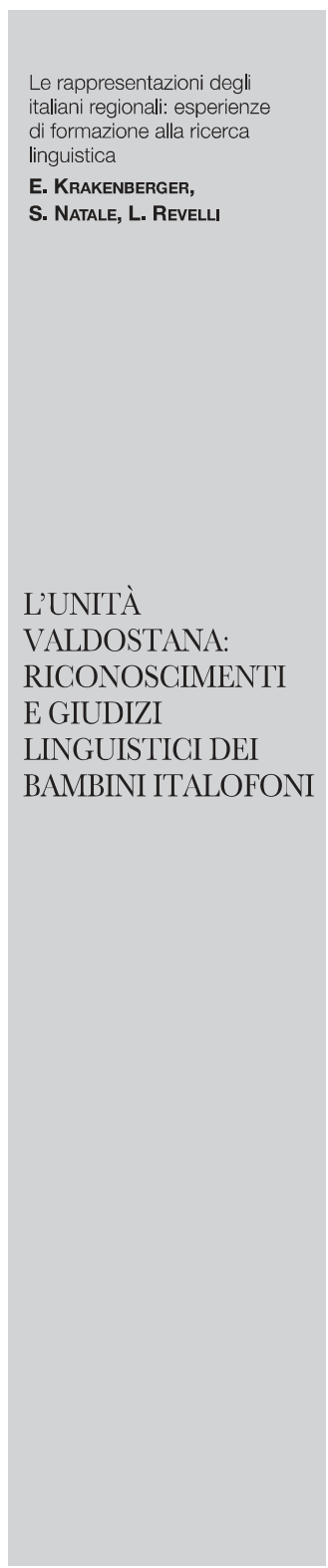

primo strumento di lavoro comune presso le due sedi, nell'incontro iniziale del Seminario agli studenti è stato parallelamente somministrato un questionario nel quale le tracce erano state disposte in una sequenza ispirata a principi di distanza fra le diverse varietà: il questionario - che per ciascun campione di italiano regionale comprendeva compiti di riconoscimento e domande volte a far emergere valutazioni sulla personalità e lo status socio-economico e culturale attribuite alle voci rappresentate nelle tracce - è stato esaminato e discusso collegialmente, quindi diffuso in forma digitale sul web tramite l'applicazione gratuita di GoogleModuli - GSuite. Nelle successive fasi di lavoro, alcune delle quali ancora in fase di svolgimento al momento della stesura di questo testo, ai duc gruppi di studenti coinvolti sono stati affidati incarichi per attività in parte comuni, in parte differenziate, come sinteticamente descritto nelle pagine che seguono.

All'Università della Valle d'Aosta partecipa alle attività seminariali un gruppo di 23 studenti iscritti al corso di laurea in Scienze della Formazione Primaria, quindi inseriti nel percorso di formazione destinato all'insegnamento nella scuola dell'infanzia e primaria. Proprio in ragione delle specificità del percorso formativo previsto, il compito prioritario dell'unità è stato identificato nell'adattamento del questionario a destinatari collocati nella fascia d'età dell'obbligo scolare. Il primo obiettivo del segmento seminariale valdostano è quindi quello di individuare nello strumento di raccolta dei dati concepito per gli adulti eventuali criticità quantitative e qualitative e di elaborare conseguenti adeguamenti per la destinazione un pubblico di parlanti di età inferiore ai 18 anni.

Una volta predisposti gli opportuni adattamenti, la nuova versione del questionario è resa raggiungibile a un indirizzo web diverso da quello originale con lo scopo di sollecitare risposte da parte di singoli parlanti o intere classi scolastiche. Le modalità di diffusione e fruizione del questionario, improntate a principi di casualità, rendono impossibile una previsione delle dimensioni e della caratterizzazione del campione ottenibile: soltanto a conclusione del periodo di raccolta sarà quindi possibile valutarne l'effettiva rappresentatività. Anche questo aspetto entrerà quindi a far parte del percorso di riflessione metodologica: gli studenti saranno, infatti, condotti a valutare criticamente l'effettiva attendibilità dei dati in relazione a diverse potenziali variabili quali le fasce d'età, le aree di provenienza, le condizioni di mono- o plurilinguismo degli informatori.

Con queste riserve, rispetto alla sezione relativa alle competenze di attribuzione delle varietà regionali a specifiche porzioni di territorio 


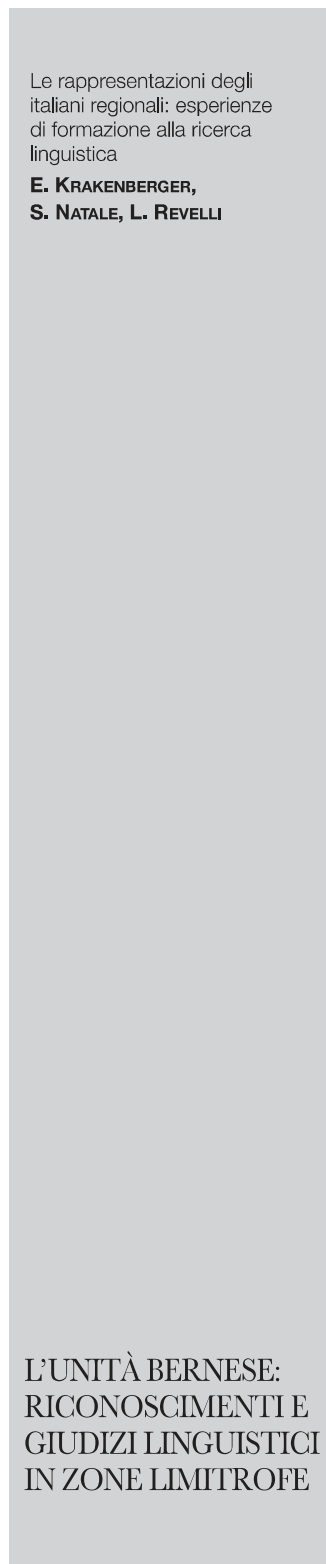

ci si proporrà di verificare in generale se e in che termini il grado di riconoscimento e percezione della variazione fonologica e prosodica progredisca con l'aumentare dell'età e, più in particolare, di controllare se sia possibile individuare una specifica fase oltre la quale la riconduzione degli accenti alle rispettive aree assume maggiore rispondenza e precisione; se tale età possa essere posta in correlazione con il crescere della conoscenza delle unità geografiche amministrative, regionali e provinciali e/o piuttosto a variabili quali la prossimità o distanza dei modelli forniti nelle tracce rispetto a quello dell' $i$ taliano regionale dei soggetti intervistati; se la collocazione nelle due aree plurilingui di confine assunte a campione (quella ticinese e appunto quella valdostana) in qualche modo predisponga o favorisca una maggiore consapevolezza delle differenze fra varietà, come alcuni studi sembrano evidenziare (Catricalà \& Di Ferrante 2011).

Per ciò che concerne gli atteggiamenti rilevabili verso le diverse varietà rappresentate nelle tracce orali, ci si proporrà di individuare eventuali fasce d'età in cui si possa collocare con buona approssimazione l'insorgenza di pregiudizi e stereotipi, tenuto conto anche del fattore prossimità/distanza geografica. In questa direzione, specifici approfondimenti saranno dedicati agli effetti epifenomenici delle pronunce straniere dell'italiano (Bianchi-Calamai 2012).

Per l'interpretazione generale dei dati raccolti attraverso i questionari un punto di riferimento di primaria importanza sarà costituito dall'indagine condotta da Giovanni Ruffino (2006) su bambini italiani di $3^{\mathrm{a}}, 4^{\mathrm{a}}$ e $5^{\mathrm{a}}$ elementare: i risultati dell'inchiesta, centrata sulla polarizzazione lingua / dialetto, conducono infatti a individuare "una precisa e già ben radicata ideologia linguistica, con la piena consapevolezza del proprio status linguistico e dei propri diversificati rapporti comunicativi, è già presente in bambini ben al di sotto dei dieci anni” (p. 55). Pur senza ambire a confermare tale assunto in relazione alle percezioni degli italiani regionali e fermo restando l'obiettivo di acquisizione di consapevolezze metodologiche piuttosto che di risultati scientificamente validati, l'unità seminariale degli studenti valdostani si proporrà, in conclusione, di tentare di verificare se una precoce consapevolezza sociolinguistica possa essere almeno ipotizzata anche in relazione alle pronunce degli italiani regionali.

L'unità bernese è composta da 12 studenti del secondo e terzo anno di bachelor del programma di studio Lingua e letteratura italiana. Oltre a condividere gli obiettivi didattici dell'unità valdostana (atteggiamenti linguistici intesi come attività di ricerca e riflessioni metodologiche), gli studenti bernesi, avvalendosi della metodologia descritta sopra, si occuperanno dell'analisi del corpus prendendo in considerazione 


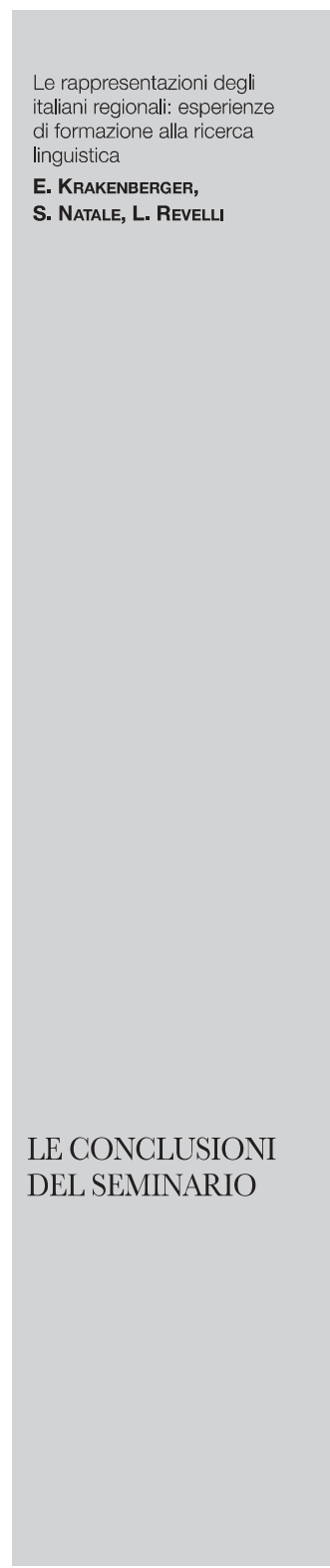

diverse variabili sociolinguistiche. Mentre l'unità valdostana si concentra sulla variabile dell' età, analizzando l'insorgenza di pregiudizi e stereotipi in bambini e ragazzi, gli studenti bernesi, lavorando con dati di informatori adulti, partono dalla domanda centrale se informatori provenienti da regioni italofone limitrofe, come il Ticino e la Valle d'Aosta, differiscono da parlanti italofoni provenienti da zone non limitrofe. In questa prospettiva, gli studenti bernesi, oltre a considerare gli atteggiamenti ottenuti in base alla provenienza degli informatori, tengono conto anche di altre varabili sociolinguistiche come sesso, grado d'istruzione e abitudini linguistiche.

Dopo una fase introduttiva, gestita dalle docenti, in cui gli studenti mediante letture approfondite hanno creato conoscenze teoriche in merito all' argomento trattato e acquisendo anche nozioni basilari per l'analisi statistica, i partecipanti del seminario hanno iniziato la fase di "laboratorio" che prevede analisi autonome. Sono state creati a tale fine tre gruppi di lavoro che hanno diviso la propria mansione per variabile sociolinguistica. Un gruppo si occupa quindi delle variabili età e sesso, un secondo gruppo considera la variabile della provenienza mentre il terzo gruppo analizza l'impatto delle abitudini linguistiche e del grado d'istruzione sul formarsi di atteggiamenti. In quest'ottica, gli studenti sono tenuti a creare delle correlazioni atte a fornire una griglia d'analisi esaustiva per poter interpretare al meglio il corpus dei dati ottenuti.

Oltre a sviluppare competenze teoriche e metodologiche, questo seminario stimola l'acquisizione di competenze interdisciplinari come la gestione del lavoro di gruppo, l'impostazione autonoma della ricerca empirica, le diverse tecniche di presentazione e il pensiero critico. La combinazione di competenze chiave variegate offre agli studenti un'opportunità per sperimentare, in un contesto guidato, le varie fasi della ricerca empirica.

Gli esiti delle indagini condotte dagli studenti delle due sedi universitarie saranno presentati il 10 dicembre 2019 in un incontro pubblico finale collocato presso la sede dell'Istituto di lingua e letteratura italiana di Berna: pur basandosi sulla presentazione dei dati raccolti, l'incontro si costituirà prioritariamente come occasione di confronto metodologico fra i due gruppi di studenti, che avranno l'occasione di discutere a proposito delle tecniche adottate, delle criticità riscontrate, dei possibili modelli interpretativi dei dati raccolti in rapporto ai modelli teorici assunti a riferimento.

Degli esiti conclusivi del Seminario si darà conto nel prossimo numero della rivista: l'auspicio è che, al di là dei risultati conseguiti rispetto allo studio del tema degli italiani regionali, l'esperienza condotta si 


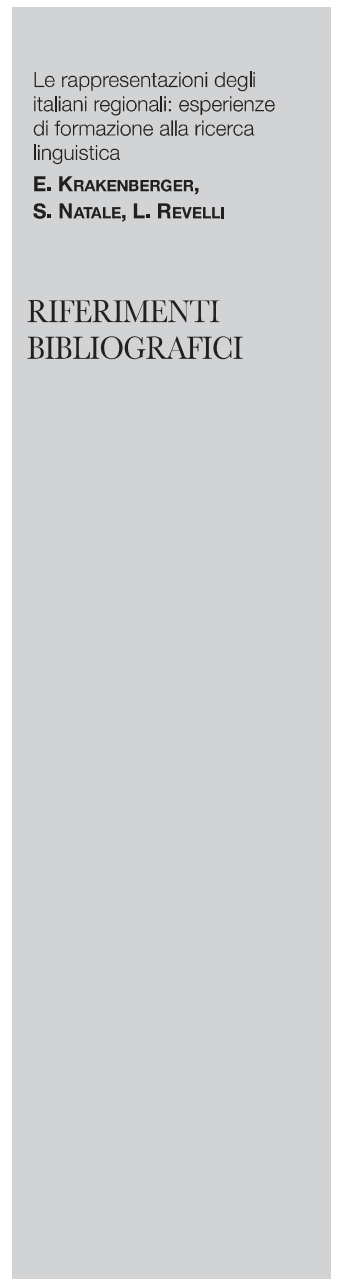

NOTA

possa configurare come opportunità di socializzazione scientifica significativa per studenti che - troppo spesso nei percorsi universitari canonici avviati a uno studio meramente teorico e individuale rischiano di sperimentare applicazioni e metodi della ricerca soltanto e nella migliore delle ipotesi in occasione della redazione della tesi di laurea, con conseguente mancata percezione della ricchezza di opportunità offerte dal lavoro in rete e sul campo.

ANTONINI F. \& MORETTI B. 2000. Le immagini dell'taliano regionale. [Locarno]: Osservatorio linguistico della Svizzera italiana.

BIANCHI F. \& CALAMAI S. 2012. Voci italiane e straniere a confronto. Indagine sugli stercotipi associati agli accenti stranicri. CAM$B I O, \mathrm{n}^{\circ} 4:$ 149-164.

CATRICALÀ M. \& DI FERRANTE L. 2011. Gli atteggiamenti verso le varietà dell'italiano: un'inchiesta di matched guise in Canton Ticino, pp. 267-287 in Moretti B., Pandolfi E.M., Casoni M. (eds). Atti del Convegno OLSI "Vitalità di una lingua minoritaria. Aspetti e proposte metodologiche”. Bellinzona: Osservatorio linguistico della Svizzera italiana.

DE PASCALE S. \& MARZO S. 2016. Gli italiani regionali. Atteggiamenti linguistici verso le varietà geografiche dell'italiano. Incontri: Rivista Europea di Studi Italiani, n³1 (1): 61-76.

RUFFINO G. 2006. L'indialetto ha la faccia scura. Giudizi e pregiudizi linguistici dei bambini italiani. Palermo: Sellerio editore.

TELMON T. 1990, Guida allo studio degli ilatiani regionali. Alessandria: Edizioni dell'Orso.

(1) Etna Krakenberger e Silvia Natale sono le docenti del Seminario presso la sede di Berna, Luisa Revelli presso la sede di Aosta. Le attività congiunte si svolgono nel quadro di un rapporto di cooperazione e mobilità (Accordo SEMP - Swirss-European Mobility Agreement) fra i due Atenei; le attività degli studenti valdostani presso la sede svizzera sono rese possibili grazie al finanziamento delle spese di viaggio da parte del CIEBP, partner organizzativo e scientifico dell'iniziativa. 